\title{
THE THEORETICAL APPROACH TO POPULATION GENETICS
}

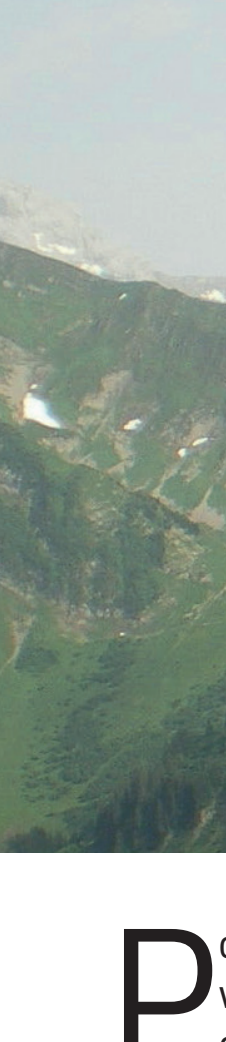

opulation genetics is the study of gene variation within or between populations. It deals with the allele frequencies in a given population and the forces, which might change this. Such forces, which are known to alter and change the allele frequency are mutation, natural selection, and genetic drift, among others. Understanding population genetics is aimed at those who have at least an intermediate knowledge of the natural sciences and a basic understanding of mathematics. It is structured around ten mathematical derivations which taken together give a deeper understanding of the theory and practice of population genetics. Well-illustrated equations supplement the text, which are helpful for understanding the concepts behind each derivation. A helpful glossary defines key scientific terms in greater detail, and chapters are accompanied by background sections, which simplify the more complex mathematics.

Säll and Bengtsson begin by discussing the effect of mutation and other forces, like genetic drift, on allele frequency before moving on to the process of selection and heterozygosity. They also provide an introduction to the use of recombination effects in measuring the association between alleles at different loci. These first three derivations are useful for large populations, however in reality not all populations quantify as large. Thankfully, the next four derivations discussed are useful for populations of a realistic size. They cover the ability to track a set of gene copies back to their origin, the role of mutation and genetic drift, the effect of both natural selection and genetic drift on mutation fixation, and the effect of genetic variation in nonhomogeneous populations. The next two derivations focus on selection, though the fixation index (FSt) which measures the differentiation time of a given subpopulation; and through the role of evolutionary forces that favour certain traits. The authors discuss the frequency-dependent selection of these traits, including how factors lead to selection which is important for organism survival, such as in the case of genes responsible for resistance against microbes, for example. The ninth derivation explores the effect of selection on quantitative traits like size, weight and length. The authors address the question of how selection will effect a trait value when it is being controlled by more than one allele. 
The book concludes with an exploration of the sex ratio as a factor of genetic system evolution under selection force. In 1858 Charles Darwin and Alfred Wallace attempted to uncover how the sex ratio for any species, which consist of male and female, is almost always close to equal. The authors skilfully outline the solution, first developed by Ronald Fisher (1930), who suggested that the random mating in any population would lead to an equal sex ratio. The mathematical proof for sex ratios that was developed in the latter half of the twentieth century is detailed here.

Understanding population genetics provides a reasoned contribution to the present field of population genetics in scientific fields such as medicine or biology. By framing the topic around the ten mathematical derivations, Säll and Bengtsson have produced a work that contributes to a deeper understanding of population genetics in both theory and practice.

\section{References}

Darwin, C. and Wallace, A. (1858) On the tendency of species to form varieties; And on the perpetuation of varieties and species by natural means of selection. Zoological Journal of the Linnean Society, 3(9), pp. 45-62, https://doi. org/10.1111/j.1096-3642.1858.tb02500.x

Fisher, R. A. (1930) The genetical theory of natural selection. Oxford: Oxford University Press. https://doi.org/10.5962/bhl.title.27468

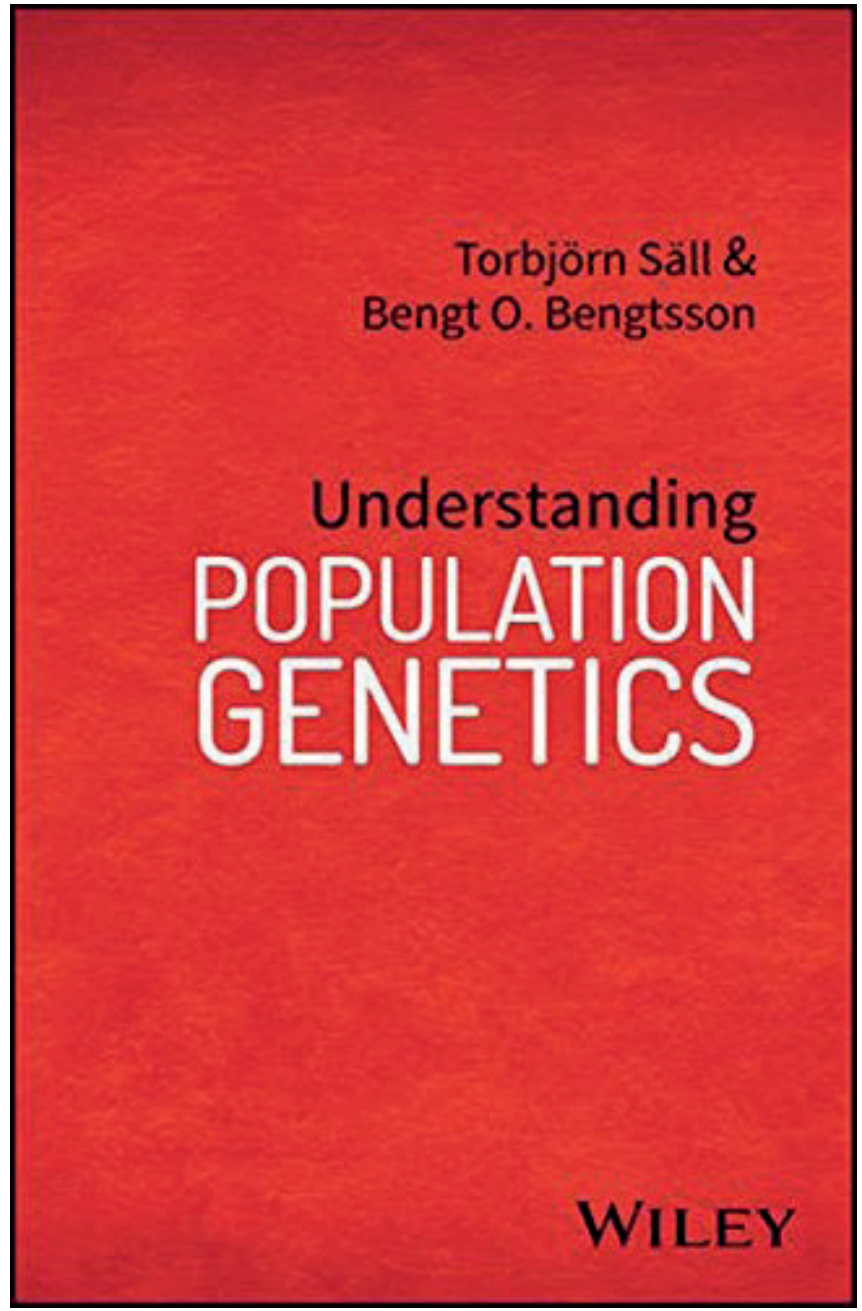

Understanding population genetics by Torbjörn Säll and Bengt O. Bengtsson (2017), Hoboken, NJ: Wiley Blackwell.

Mataab K. Al Ghafri is a PhD candidate at Edinburgh Napier University and works for the Office for Conservation of Environment (OCE) in Oman. His research focuses on the conservation, population status and effect of isolation on the gene pool of the Nubian ibex (Capra nubiana) and the relatedness to other populations across the species range. 